\title{
A STUDY ON GENETIC DIVERGENCE OF THE RED SQUIRREL SCIURUS VULGARIS (RODENTIA: MAMMALIA) FROM SIX REGIONS IN EURASIA: BASED ON CYTOCHROME B COMPLETE SEQUENCES
}

\author{
Hung Sun $\mathrm{Koh}^{1 *}$, Damdingiin Bayarkhagva ${ }^{2}$, Alexey Kryukov ${ }^{3}$ \\ Minghai Zhang ${ }^{4}$, Bae Kun Lee ${ }^{1}$, Dava Bazarsad ${ }^{2}$ and Sin Wan Ho ${ }^{1}$ \\ ${ }^{1}$ Research Institute for Biological Resources, Sejong 339-941, Korea \\ ${ }^{*}$ E-mail: syskoss@chungbuk.ac.kr \\ ${ }^{2}$ Department of Molecular Biology, National University of Mongolia \\ Ulaanbaatar 210646, Mongolia; E-mail: dambayar@yahoo.com \\ ${ }^{3}$ Laboratory of Evolutionary Zoology and Genetics, Far East Division \\ Russian Academy of Sciences, Vladivostok 690022, Russia; E-mail: kryukov@ibss.dro.ru \\ ${ }^{4}$ College of Wildlife Resources, Northeast Forestry University, Harbin 150040, China \\ E-mail: Zhangminghai2004@126.com
}

\begin{abstract}
Cytochrome $b$ complete sequences $(1140 \mathrm{bp})$ of Sciurus vulgaris from four regions in continental Asia (Korea, northeastern China, far-eastern Russia, and Mongolia) were obtained and analysed with corresponding complete and partial (359 bp) haplotypes of S. vulgaris, obtained from GenBank. These sequences were used to compare our and previous results from partial sequences and to examine population differentiation of $S$. vulgaris in Eurasia by using complete sequences and by using more numerous specimens. Based on partial sequences from ten Eurasian regions (the four regions in Asia, Japan, Portugal, western China, Russia, and northern and southern Italy), we found that S. vulgaris is composed of two clades (a minor one from southern Italy and a major one from other Eurasian regions). We considered that our results are more conclusive than previous results by other researchers, performed without using haplotypes from Korea, Mongolia, and far-eastern Russia and detected three clades (southern Italy, Russia, and other Eurasia) or two clades with one distinguishable haplotype. Moreover, we found that our results from partial sequences are less informative than those from complete sequences, indicating that cytochrome $b$ complete sequences from Eurasia are needed in order to examine population structure of S. vulgaris. Finally, in this study on population differentiation from cytochrome $b$ complete sequence analyses, we found that genetic divergence of six Eurasian populations, including the four Asian populations, is low, and that our results do not support the current subspecies classification, recognizing 23 subspecies.
\end{abstract}

Key words: Cytochrome $b$, Sciurus vulgaris, Eurasia, phylogeography, DNA systematics.

\section{INTRODUCTION}

The Red squirrel (Sciurus vulgaris Linneaus, 1758) occurs in wooded areas of most of the Palaearctic, with 23 subspecies being recognized (THORINGTON \& HofFmann 2005). Moreover, Jones and Johnson (1965) reported that the Red squirrel from Korea (S. v. coreae) was different from the Red squirrel from north- 
eastern China (S. v. mamtchuricus) in the external and cranial morphology. On the other hand, Corbet (1978) recognized only two subspecies within S. vulgaris (S. v. vulgaris from continental Eurasia and insular Sakhalin and Hokkaido and S. v. leucourus from insular England and Ireland). WAUters and MARTINOLI (2008) also proposed a new subspecies for the Red squirrel in southern Italy, indicating that subspecies classification of $S$. vulgaris is still controversial.

Nuclear genes with a slower rate of evolution are useful for phylogeny construction at a higher level, whereas mitochondrial DNA (mtDNA) is suitable for classification at the species level and the examination of population structure within a species (SUNNUCKs 2000). Phylogenetic relationships among the six species in the genus Sciurus were inferred from cytochrome $b$ partial sequences of $1040 \mathrm{bp}$ (Oshida \& Masuda 2000), and the relationships among the seven species in Sciurus were analysed from cytochrome $b$ complete sequences (Oshida et al. 2009).

In S. vulgaris genetic divergence within the Italian Alps was examined on the basis of control region partial sequences (378 bp) and microsatellites (Trizio et al. 2005). They reported that both nuclear and mtDNA data indicate a significant differentiation among study sites and a significant correlation between genetic and geographical distance only over a large scale. Three clades (southern Italy, Russia, and other Eurasia) were detected based on partial sequences ( $252 \mathrm{bp}$ ) of control region and partial sequences ( $359 \mathrm{bp}$ ) of the cytochrome $b$ gene (Liv et al. 2014). However, GriLl et al. (2009) found two clades (southern Italy and other Eurasia), although one Russian haplotype was distinguishable basally to the European group, with high bootstrap support. On the other hand, lack of differentiation between adjacent populations of $S$. vulgaris were revealed from control region partial sequences (395 bp) of Ireland and England (FINNEGAN et al. 2008) and from control region complete sequences (1058 bp) of Korea and northeastern China (Кон et al. 2006).

We can expect to obtain better information from the results based on cytochrome $b$ complete sequences and more numerous geographic locations, compared to the results on the basis of cytochrome $b$ partial sequences and fewer geographic locations. Additionally, Liv et al. (2014) stated that a more extensive study with Eurasian specimens is necessary to draw more determinate conclusions on phylogeographical history of $S$. vulgaris. Thus, a sequencing study with complete sequences of the cytochrome $b$ gene from other Asian regions, such as Korea, far-eastern Russia, and Mongolia, is necessary to examine genetic differentiation among Asian populations and Eurasian populations of S. vulgaris. However, this portion of the research has not been performed yet.

In this paper, 21 cytochrome $b$ complete haplotypes $(1140 \mathrm{bp})$ of $S$. vulgaris from four populations in continental Asia (Korea, northeastern China, fareastern Russia, and Mongolia) were obtained and compared to five complete haplotypes of S. vulgaris from Japan and Portugal, obtained from GenBank, 
in order to examine genetic differentiation among Eurasian populations. In addition, 26 partial haplotypes (359 bp) of $S$. vulgaris, obtained from the 26 complete haplotypes, were analysed with six partial haplotypes of $S$. vulgaris from four other regions in Eurasia (western China, Russia, southern Italy, and northern Italy), obtained from GenBank, to compare our and previous results from partial sequences.

\section{MATERIAL AND METHODS}

We used Sherman live traps and collected 29 specimens of $S$. vulgaris from 16 locations in the four Asian regions, as given in Table 1. The 16 collection sites are also shown in Figure 1. We anesthetized and killed animals with chloroform. Small pieces of muscle

Table 1. Location, specimen number, and cytochrome $b$ haplotypes of Sciurus vulgaris, used in this study. Twenty-one cytochrome $b$ complete haplotypes (1140 bp) were obtained from 29 specimens at 16 locations of four regions (Korea, northeastern China, far-eastern Russia, and Mongolia) in Asia, as shown in Figure 1.

\begin{tabular}{|c|c|}
\hline Location & Specimen number (cytochrome $b$ haplotype) \\
\hline \multicolumn{2}{|l|}{ Korea } \\
\hline Mt. Songri & KRS011 (Sg01Wl01) \\
\hline Mt. Weolak & KRS004 (Sg01W101), KRS005 (W102) \\
\hline Mt. Sobaek & KRS012 (Sb01); KRS021 and KRS022 (Sb02) \\
\hline \multicolumn{2}{|l|}{ Northeastern China } \\
\hline Harbin & CRS006 (Hb01), CRS007 (Hb02), CRS008 (Hb03) \\
\hline Ichun & CRS011 (Ic01), CRS002 (Ic02) \\
\hline \multicolumn{2}{|l|}{ Far-eastern Russia } \\
\hline Vladivostok & RRS597 and RRS598 (V101) \\
\hline Chuguevsky District & RRS2600 (Cg01) \\
\hline Anuchinsky District & RRS2601 (Ac01) \\
\hline \multicolumn{2}{|l|}{ Mongolia } \\
\hline Ulaanbaatar & MRS001 (U101) \\
\hline Khentii Province & MRS002 (Kt01) \\
\hline Selenge Province & MRS003 (S101Bg01Kv01By01) \\
\hline Bulgan Province & MRS004 (Bg02), MRS005 (S101Bg01Kv01By01) \\
\hline Khovsgol Province & $\begin{array}{c}\text { MRS007 (Kv02), MRS008 (Kv03), MRS009 (Kv04), MRS012 } \\
\text { (Sl01Bg01Kv01By01) }\end{array}$ \\
\hline Zavkhan Province & MRS006 and MRS011 (Ak01Zk01), MRS015 (Zk02) \\
\hline Arkhangai Province & MRS013 (Ak01Zk01) \\
\hline Bayan-Oligii Province & MRS014 (S101Bg01Kv01By01) \\
\hline
\end{tabular}


Table 2. Location and accession number of Sciurus vulgaris, obtained from GenBank. Five cytochrome $b$ complete haplotypes $(1140 \mathrm{bp}$ ) from Eurasia (three from Japan in Asia and two from Portugal in southwestern Europe), and six partial haplotypes (359 bp) from Eurasia (two from northern Italy and one from southern Italy in southern Europe, one from Russia, and two from western China) were used for this study, and partial haplotypes were indicated by asterisks.

\begin{tabular}{lll}
\hline Region & Location & GenBank accession number \\
\hline Japan & Hokkaido & AB292679, AB292680, AB292681 \\
SW Europe & Portugal & JX457817, JX457818 \\
S Europe & Verese, northern Italy & FJ932497* \\
& Belluno, northern Italy & FJ932504* \\
& Calabria, southern Italy & FJ932489* \\
& Russia & FJ932488 \\
E Europe & Altai Mountains & JX482613* \\
W China & Tianshan Mountains & JX482619* \\
& &
\end{tabular}

tissues then were preserved in a deep freezer at $-60^{\circ}$. Total cellular DNA was extracted using a genomic DNA extraction kit (Intron Co., Daejeon, Korea). The cytochrome $b$ gene was PCR-amplified using primers L14724 and H15915, designed by IRwin et al. (1991). The PCR thermal cycle employed was as follows: $94^{\circ}$ for $5 \mathrm{~min} ; 94^{\circ}$ for $1 \mathrm{~min}, 60^{\circ}$ for $1 \mathrm{~min}, 72^{\circ}$ for $1 \mathrm{~min}$ ( 32 cycles); and $72^{\circ}$ for $5 \mathrm{~min}$. To remove primers and unincorporated nucleotides, the amplified products were purified using a DNA PrepMate kit with a silica-based matrix

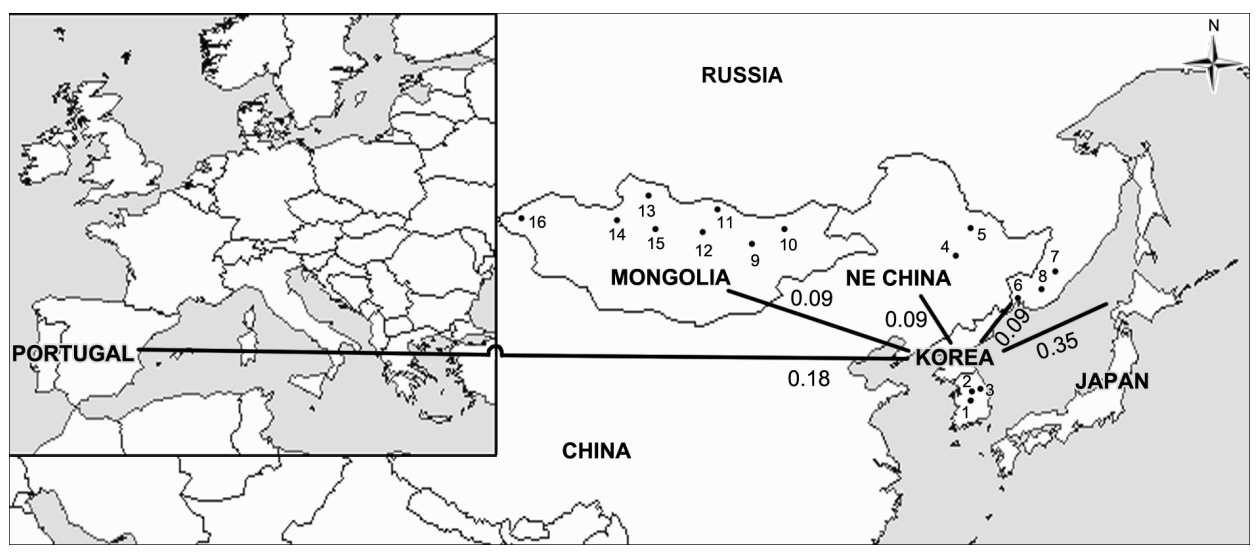

Fig. 1. Collection sites for 29 Sciurus vulgaris specimens from 16 locations of four regions in continental Asia, used in this study. Three locations from Korea (1-3), two locations in northeastern China (4 and 5), three locations in far-eastern Russia (6-8), and eight locations from Mongolia (9-16) are listed in Table 1, with number of specimens in each location. Minimum Jukes-Cantor distances (\%) between four haplotypes from Korea and 22 haplotypes from five other regions (northeastern China, far-eastern Russia, Mongolia, Japan, and Portugal) in cytochrome $b$ complete sequences are given above the lines, connecting Korea and five other regions 
(Intron Co., Korea). For sequencing, the purified PCR products were analysed with an automated DNA Sequencer (Perkin Elmer 377) at Bioneer Co. (Seoul, Korea).

Cytochrome $b$ complete sequences (1140 bp) were obtained from 29 specimens of $S$. vulgaris in the four Asian regions, as listed in Table 1, and these sequences were compared to five complete haplotypes of S. vulgaris from Japan and Portugal, obtained from GenBank, as given in Table 2. It should be noted that although sequences in each of the three haplotypes from Portugal were $1128 \mathrm{bp}$, we considered them as complete sequences. In addition, partial sequences ( $359 \mathrm{bp}$; site nos. 85 to 443 ) were obtained from the complete sequences, and these partial sequences were compared to the six partial sequences from four other regions in Eurasia, obtained from GenBank, as listed in Table 2.

Sequence alignment, detection of parsimonious informative sites, model selection, calculation of nucleotide distances, and tree constructions with 1000 bootstrapped replications were conducted using MEGA5 (TAmura et al. 2011). The Jukes-Cantor (JC) model, which showed the lowest Bayesian information criterion score, was chosen as the best model for our data by the program. Maximum likelihood trees were constructed by the JC model. Tamias senex (AF147665) and Sciurus lis (AB192923) were used as outgroups.

Ethics statement: No governmental permission is required for collecting Red squirrel (Sciurus vulgaris) in Korea, China, Russia, and Mongolia for this kind of scientific study.

\section{RESULTS}

From 29 cytochrome $b$ complete sequences (1140 bp) of $S$. vulgaris in the four regions of Asia, 21 haplotypes were identified, as given in Table 1. Within 26 cytochrome $b$ complete haplotypes of $S$. vulgaris ( 21 haplotypes obtained from this study, and five haplotypes obtained from GenBank), 33 sites (2.81\%) were variable, and eight sites $(0.70 \%)$ were parsimony informative.

A maximum likelihood tree with the 26 cytochrome $b$ complete haplotypes of S. vulgaris is shown in Figure 2. The 26 haplotypes from the six regions in Eurasia (Gp 1, Korea; Gp 2, northeastern China; Gp 3, far-eastern Russia; Gp 4, Mongolia; Gp 5, Japan; and Gp 6, Portugal in southwestern Europe) were not divided into any subgroups, and a bootstrap value supporting all 26 haplotypes was 100. Average JC distances among the four populations in continental Asia (Gps 1 to 4 ) ranged from $0.20 \%$ to $0.26 \%$, with the same ranges revealed among the five populations in continental Eurasia (Gps 1 to 4 and Gp 6). On the other hand, average JC distance between insular the Japanese population (Gp 5) and five other populations in continental Eurasia (Gps 1 to 4 and Gp 6) was $0.41 \%$. Additionally, three haplotypes from three regions in Asia ( $\mathrm{Hb} 02$ from Harbin in northeastern China, Vl01 from Vladivostok in far-eastern Russia, and Sl1Bg1Kv1By1 from four Provinces in Mongolia) were identical in their sequences. Minimum JC distances between four haplotypes from Korea and 22 haplotypes from five other Eurasian populations (northeastern China, far-eastern Russia, Mongolia, Japan, and Portugal) were $0.09 \%$, $0.09 \%, 0.09 \%, 0.35 \%$, and $0.18 \%$, respectively, as given in Figure 1 . 


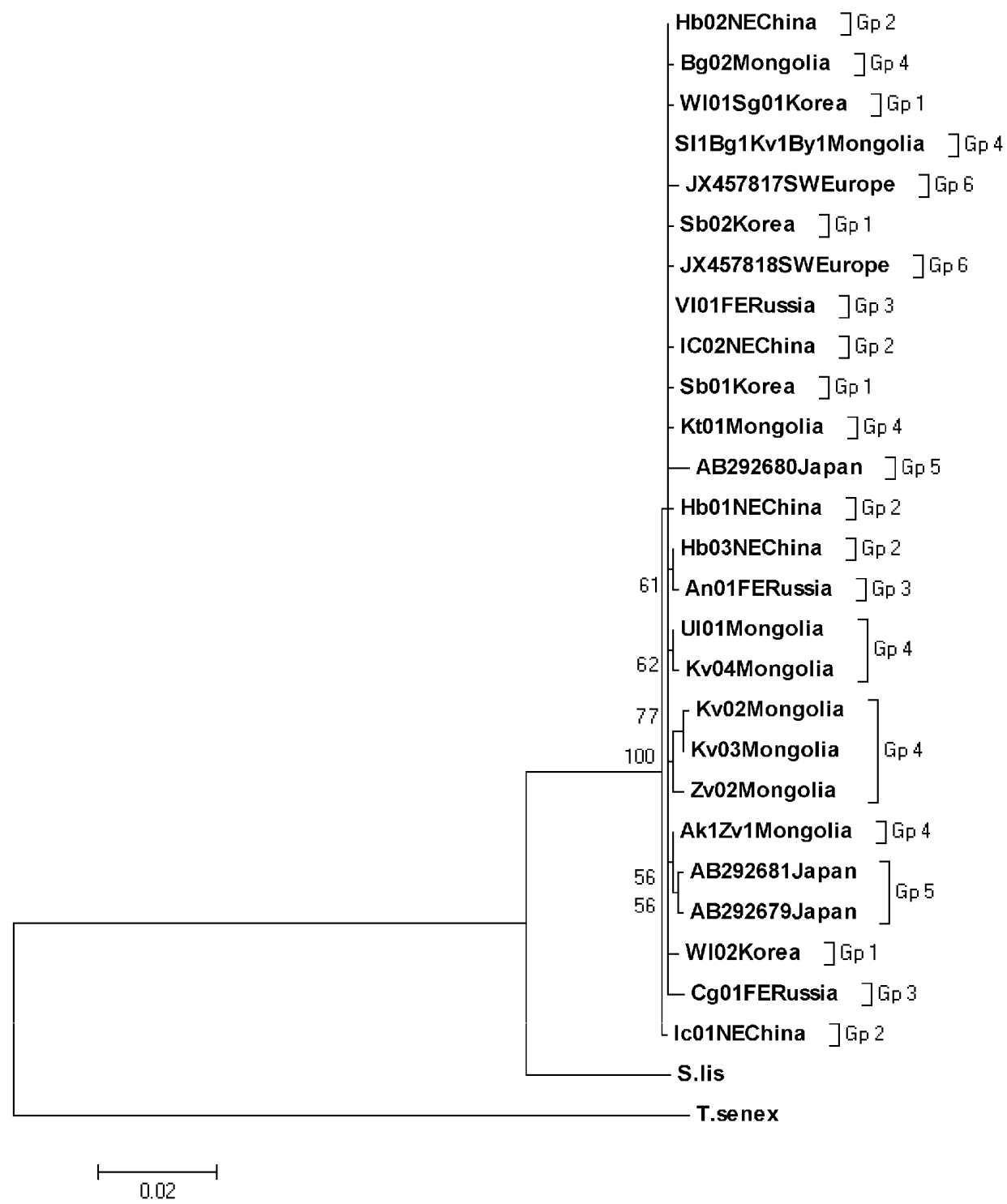

Fig. 2. A maximum likelihood tree with 26 cytochrome $b$ complete haplotypes (1140 bp) from Sciurus vulgaris. Twenty-one haplotypes from Korea, northeastern China, far-eastern Russia, and Mongolia were obtained from this study, as listed in Table 1, and location name follows haplotype name in each haplotype. Five other haplotypes from Japan and Portugal were obtained from GenBank, and location name follows accession number in each haplotype. The tree was constructed with 1000 bootstrapped replications, and the bootstrap values $>50 \%$ are reported at the internodes. Tamias senex (AF147665) and Sciurus lis (AB192923) were used as outgroups 


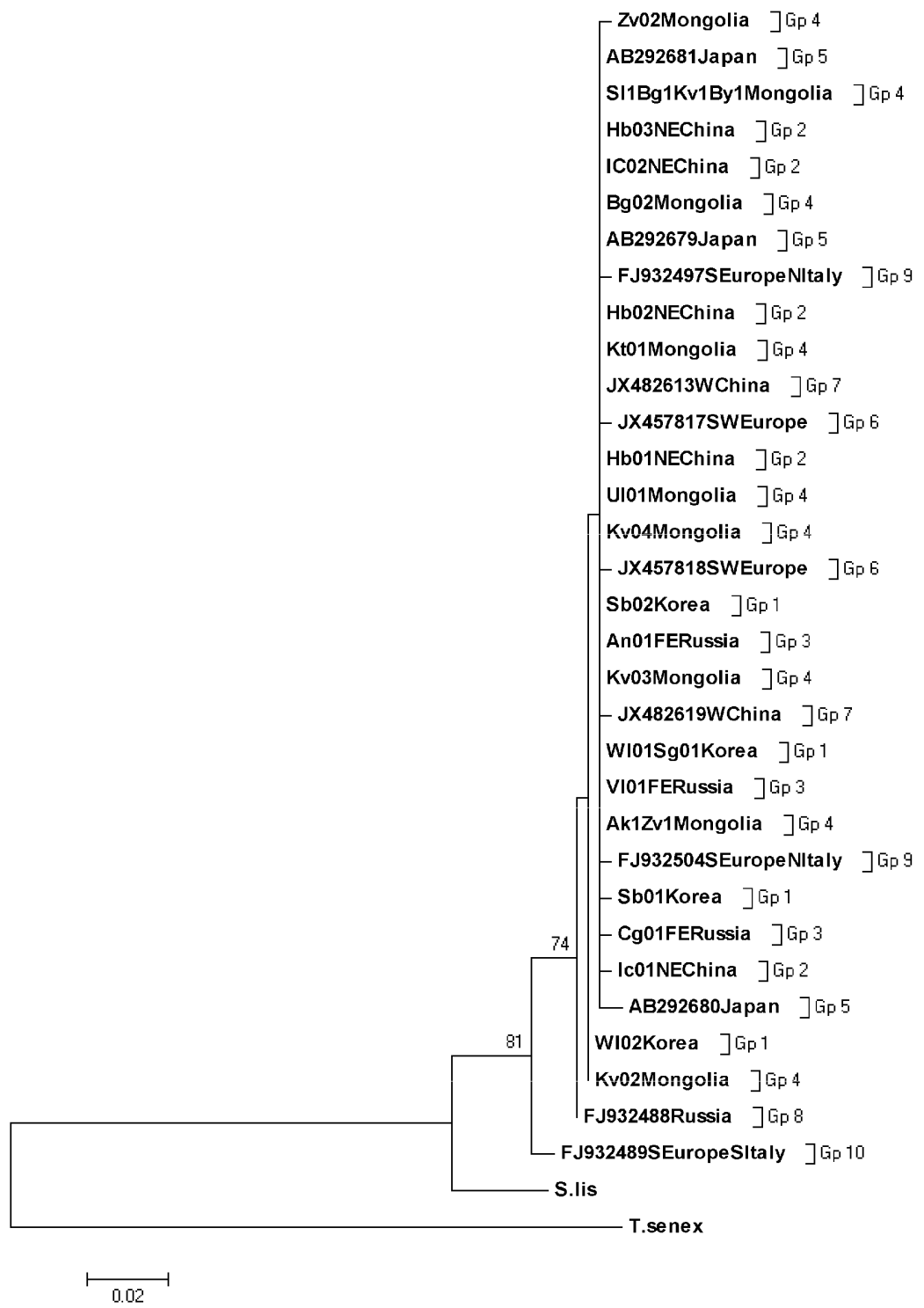

Fig. 3. A maximum likelihood tree with 32 cytochrome $b$ partial haplotypes (359 bp) from Sciurus vulgaris. Twenty-six haplotypes from Korea, northeastern China, far-eastern Russia, and Mongolia were obtained from complete sequences, as listed in Tables 1 and 2, and location name follows haplotype name in each haplotype. Six other haplotypes from northern and southern Italy, Russia, and western China were obtained from GenBank, and location name follows accession number in each haplotype. The tree was constructed with 1000 bootstrapped replications, and the bootstrap values $>50 \%$ are reported at the internodes. Tamias senex (AF147665) and Sciurus lis (AB192923) were used as outgroups 
Within 32 cytochrome $b$ partial (359 bp) haplotypes (26 partial haplotypes, obtained from complete sequences, and six partial haplotypes, obtained from GenBank), 18 sites (5.01\%) were variable, and two sites $(0.56 \%)$ were parsimony informative. A maximum likelihood tree with the 32 cytochrome $b$ partial haplotypes of $S$. vulgaris from ten populations in Eurasia (Gps 1 to 10) is shown in Figure 3. Two clades were detected: a minor clade of one haplotype in southern Italy (Gp 10) and a major clade from 31 haplotypes in other Eurasia (Gp 1, Korea; Gp 2, northeastern China; Gp 3, far-eastern Russia; Gp 4, Mongolia; Gp 5, Japan; Gp 6, Portugal; Gp 7, western China; Gp 8, Russia; and Gp 9, northern Italy), with average JC distance of $2.33 \%$ (range, $1.69 \%$ $2.84 \%$ ) and six fixed site differences (site nos. $87,191,219,315,342$, and 417 ). A bootstrap value supporting all 32 partial haplotypes (the two clades) was 81 , and average JC distance among the 31 partial haplotypes (the major clade) was $0.24 \%$ (range, $0.00 \%-0.84 \%$ ).

Additionally, within the major clade (31 haplotypes) 18 partial haplotypes from the six regions in Asia (Korea, northeastern China, western China, Mongolia, far-eastern Russia, and Japan) were identical in their sequences, and a bootstrap value supporting the 31 partial haplotypes was 74 . Two other partial haplotypes from Korea and Mongolia (W102 and Kv02, respectively) were also identical in their sequences, and they were located between one Russian haplotype (Gp 8, FJ932488) and 28 other haplotypes from other Eurasia (Gps 1 to 7 and Gp 9) within the major clade.

\section{DISCUSSION}

From mtDNA partial control region and cytochrome $b$ haplotypes LiU et al. (2014) concluded that $S$. vulgaris is composed of three clades (southern Italy, Russia, and other Eurasia), although they detected each of the two clades (southern Italy and Russia) from only one haplotype. On the other hand, GrILL et al. (2009) found two clades (southern Italy and other Eurasia), despite the Russian clade by Liu et al. (2014) being distinguishable from the other Eurasian clade, with high bootstrap support. Moreover, these studies did not utilize the specimens from Korea, Mongolia, and far-eastern Russia. In this analysis with the 32 cytochrome $b$ partial haplotypes from Eurasia, including our data from Korea, northeastern China, far-eastern Russia, and Mongolia (Fig. 3), we newly found that S. vulgaris is composed of two clades: a minor one from single haplotype in southern Italy (Gp 10) and a major one from 31 haplotypes in other Eurasia (Gps 1 to 9), with average JC distance of 2.33\% (range, 1.69\%-2.84\%) and six fixed site differences between the two clades. It should be noted that in our study one Russian haplotype (Gp 8, FJ932488), being recognized as a distinguishable haplotype by GriLl et al. (2009) and a 
distinct clade by Liu et al. (2014), was clustered together with two haplotypes from Korea and Mongolia (Wl02 and Kv02) and 28 other haplotypes from Eurasia (Gps 1 to 7 and Gp 9), which formed the major clade.

Additionally, Grill et al. (2009) and Liu et al. (2014) failed to get a bootstrap value supporting the three clades of $S$. vulgaris, but in our analysis (Fig. 3 ) we first found that a bootstrap value supporting all 32 partial haplotypes of the two clades is 81 (Fig. 3). However, GrILl et al. (2009) reported that a bootstrap value supporting the two clades (Russia and other Eurasia) was 93, but they have only used six haplotypes with identical sequences from southern Italy in their analyses. Thus, we considered that our results from the 32 partial haplotypes, including the 21 haplotypes from Korea, northeastern China, fareastern Russia, and Mongolia, newly obtained by us, are more informative than the previous results from partial sequences by GriLl et al. (2009) and LiU et al. (2014).

From our partial sequence analysis of S. vulgaris (Fig. 3), we found that a bootstrap value supporting 31 haplotypes of the major clade is 74 , but based on complete sequence analysis (Fig. 2) we recognized that a bootstrap value supporting all 26 haplotypes was 100 . We also found that among 32 partial haplotypes, 18 partial haplotypes from six regions in Asia (Korea, northeastern China, far-eastern Russia, Mongolia, Japan, and western China) and two haplotypes from Korea and Mongolia showed identical sequences (Fig. 3), whereas among 26 complete haplotypes, three complete haplotypes from northeastern China, far-eastern Russia, and Mongolia were identical, as shown in Figure 2. We found that the results from partial sequences are not conclusive to determine genetic diversity and population structure of $S$. vulgaris, compared to the results from complete sequences. Thus, we concluded that complete sequences of the cytochrome $b$ gene from Eurasia are needed in order to study on population differentiation of $S$. vulgaris.

Moreover, in this study (Fig. 2) we used complete sequences of the cytochrome $b$ gene, which was not utilized by former researchers in order to examine genetic diversity of $S$. vulgaris from Eurasia. We did not obtain complete sequences of the cytochrome $b$ gene from the specimens across Europe, especially southern Italian specimens, but, for further discussions in this study we used our results from complete sequences.

Central populations of a species range are relatively homogeneous (ЕскERT et al. 2008), and a genetic distance of $<2 \%$ based on the cytochrome $b$ gene was typical of population and intraspecific variation (BRADLEY \& BAKER 2001). Asian populations have been shown to display this homogeneity in the Asian lesser white-toothed shrew (Crocidura shantungensis) and the striped field mouse (Apodemus agrarius) (Кон et al. 2013, 2014). 
In this analysis (Fig. 2) based on 26 cytochrome $b$ complete haplotypes of S. vulgaris from the six Eurasian regions, we found that the range of average JC distances among the four populations in continental Asia (Gps 1 to 4) and that among the five populations in continental Eurasia (Gps 1 to 4 and Gp 6) are the same, with the range from $0.20 \%$ to $0.26 \%$. Additionally, three haplotypes from northeastern China, far-eastern Russia, and Mongolia showed identical sequences. Thus, we concluded that genetic diversity among five continental Eurasian populations of $S$. vulgaris is low.

Island populations should diverge over time (morphologically and genetically) from populations of respective mainland species (JoHnson et al. 2000). Although at the end of the last glacial period, large areas of continental shelf was dry land, allowing easy exchange of plant and animals by land bridge connections to what are now isolated islands (Lomolino et al. 2010). From 26 cytochrome $b$ complete sequences of $S$. vulgaris (Fig. 2) average JC distance between insular Japanese population (Gp 5) and five other populations in continental Eurasia (Gps 1 to 4 and Gp 6) was $0.41 \%$, indicating that the Japanese population has been connected to adjacent continental populations in far-eastern Russia during the last glacial period.

Peripheral populations are often genetically and morphologically divergent from central populations, and natural barriers to dispersal, which limit species distribution, include mountain range and rivers (Goldberg \& LAND 2007). However, peninsular effects appear to be idiosyncratic, depending on context, taxa, scales and taxonomic levels (BAттіsтi 2014). Northern boundary of the Korean Peninsula was formed naturally by Yalu River, Baitou Mountain (the main peak of the Changbai Mountains in northeastern China, 2744 $\mathrm{m}$ above sea level), and Tuman River. Minimum JC distances between four haplotypes from Korea (Gp 1) and 17 haplotypes from five other Eurasian populations (Gps 2 to 6: northeastern China, far-eastern Russia, Mongolia, Japan, and Portugal) were $0.09 \%, 0.09 \%, 0.09 \%, 0.35 \%$, and $0.18 \%$, respectively, as shown in Figure 1. Thus, we considered that the northern boundary of the Korean Peninsula did not play a role for a natural barrier between S. ulgaris in the Korean Peninsula and that in other continental Eurasia. We also concluded that genetic diversity among Eurasian six populations of $S$. vulgaris is also negligible.

Additionally, BAtтisti et al. (2013) noted that rapid range expansion occurred in S. vulgaris in southern Italy, based on the data from distribution history. GRILL et al. (2009) and Liu et al. (2014) also noted that S. vulgaris has expanded its range rapidly from a relatively small founding population in southern Italy, on the basis of mtDNA control region and cytochrome $b$ partial haplotypes. From cytochrome $b$ complete sequences (Fig. 2), we found negligible genetic divergence in the six Eurasian populations of $S$. vulgaris, and we 
considered that the low genetic diversity of these Eurasian populations is due to rapid range expansion after the last glacial period. However, we could not discuss on the ancestral population of $S$. vulgaris because we could not obtain cytochrome $b$ complete haplotypes from southern Italy and Russia.

A subspecies is an aggregate of phenetically similar populations of a species differing taxonomically from other populations of that species (MAYR \& AsHLOck 1991). It was advocated that a classification should reflect all available characters distributed as widely and as evenly as possible over the organisms studied (HuELSENBECK et al. 1996). In our analyses from 26 cytochrome $b$ complete haplotypes of $S$. vulgaris, performed without using southern Italian and Russian haplotypes (Fig. 2), population subdivisions were not detected, and we could not recognize the 23 subspecies. As a result, we considered that our results do not to support the current subspecies classification by THORINGTON and HoFFMANN (2005).

\section{REFERENCES}

Batтisti, C. (2014) Peninsular patterns in biological diversity: historical arrangement, methodological approaches and causal processes. Journal of Natural History 48: 2701-2732. doi: 10.1080/00222933.2014.925599

Battisti, C., Amori, G., Angelici, F. M., Capizzi, D., De Felici, S., Mortelliti, A., Schiavano, A., Verucci, P. \& Zapparoli, M. (2013) Evidence of a local range expansion in a fragmentation-sensitive species: the case of red squirrel (Sciurus vulgaris) in central Italy. Vie et milieu - Life and Environment 63: 59-65.

Bradley, R. D. \& BAKer, R. J. (2001) A test of the genetic species concept: cytochrome $b$ sequences and mammals. Journal of Mammalogy 82: 960-973. doi: 10.1644/15451542(2001)082<0960:ATOTGS $>2.0 . C O ; 2$

Corbet, G. B. (1978) The mammals of the Palaearctic region: a taxonomic review. British Museum (Natural History), Cornell University Press, London, 314 pp.

Eckert, C. G., Samis, K. E. \& Lougheed, C. (2008) Genetic variation across species' geographical ranges: the central-marginal hypothesis and beyond. Molecular Ecology 17: 1170-1188. doi: 10.1111/j.1365-294X.2007.03659.x

Finnegan, L. A., Ceiridwen, J. E. \& John, M. R. (2008) Origin of, and conservation units in, the Irish red squirrel (Sciurus vulgaris) population. Conservation Genetics 9: 1099-1109. doi: 10.1007/s10592-007-9419-7

Goldberg, E. E. \& Land, L. (2007) Species and dispersal barriers. The American Naturalist 170: 297-304. doi: 10.1086/518946

Grill, A., Giovanni, A., Gaetano, A., Irene, L., Guido, T., Lucas, A. W. \& Ettore, R. (2009) Molecular phylogeography of European Sciurus vulgaris: refuge within refugia. Molecular Ecology 18: 2687-2699. doi: 10.1111/j.1365-294X.2009.04215.x

Huelsenbeck, J. P., Bull, J. J. \& Cunningham, C. W. (1996) Combining data in phylogenetic analysis. Trends in Ecology and Evolution 11: 152-157. doi: 10.1016/0169-5347(96)10006-9

Irwin, D. M., Kocher, T. D. \& Wilson, A. C. (1991) Evolution of the cytochrome $b$ gene of mammals. Journal of Molecular Evolution 32: 128-144. doi: 10.1007/BF02515385 
Johnson, K. P., Adler, F. R. \& Cherry, J. L. (2000) Genetic and phylogenetic consequences of island biogeography. Evolution 54: 387-396. doi: 10.1111/j.0014-3820.2000.tb00041.x

Jones, J. K. \& Johnson, D. H. (1965) Synopsis of the lagomorphs and rodents of Korea. University of Kansas Publication, Museum Natural History 16: 357-407.

Koн, H. S., Zhang, M., WANG, J., Shin, D. S. \& Lee, B. G. (2006) Mitochondrial DNA variation in the red squirrel (Sciurus vulgaris) from Korea and northeastern China. Acta Zoologica Sinica 26: 1-7.

Koh, H. S., Kartavtseva, I. V., Lee, B. K., Kweon, G. H., Yang, B. G., Heo, S. W. \& In, S. T. (2013) A preliminary study on genetic diversity of the Asian lesser white-toothed shrew Crocidura shantungensis (Mammalia: Soricomorpha) in mainland Korea, adjacent islands, and continental East Asia: cytochrome $b$ sequence analyses. Russian Journal of Theriology 12: 71-77.

Koh, H. S., Shaner, P. J., Csorba, G., Wang, Y., Jang, K. H. \& Lee, J. H. (2014) Comparative genetics of Apodemus agrarius (Rodentia: Mammalia) from insular and continental Eurasian populations: cytochrome $b$ sequence analyses. Acta Zoologica Academiae Scientiarum Hungaricae 60: 73-84.

Liu, Z., Li, B., MA, J., Zheng, D. \& Xu, Y. (2014) Phylogeography and genetic diversity of the red squirrel (Scuirus vulgaris) in China: implications for the species postglacial expansion history. Mammalian Biology 79: 247-253. doi: 10.1016/j.mambio.2014.02.004

Lomolino, M. V., Riddle, B. R., Whittaker, R. J. \& Brown, J. H. (2010) Biogeography. 4th ed. Sinauer Associates, Sunderland, MA.

Mayr, E. \& Ashlock, P. D. (1991) Principles of systematic zoology. McGraw-Hill, New York, $475 \mathrm{pp}$

Oshida, T. \& Masuda, R. (2000) Phylogeny and zoogeography of six squirrel species of the genus Sciurus (Mammalia, Rodentia), inferred from cytochrome $b$ gene sequences. Zoological Science 17: 405-409. doi: 10.2108/zsj.17.405

Oshida, T., Arslan, A. \& NodA, M. (2009) Phylogenetic relationships among the old world Sciurus squirrels. Folia Zoologica 58: 14-25.

Sunnucks, P. (2000) Efficient genetic markers for population biology. Trends in Ecology and Evolution 15: 199-203. doi: 10.1016/S0169-5347(00)01825-5

Tamura, K., Peterson, D., Peterson, N., Stecher, G., Nei, M. \& Kumar, S. (2011) Mega5: Molecular evolutionary genetics analysis using maximum likelihood, evolutionary distance, and maximum parsimony methods. Molecular Biology and Evolution 28: 2731-2739. doi: 10.1093/molbev/msr121

Thorington, R. W. \& Hoffmann, R. S. (2005) Order Rodentia. In: Wilson, D. E. \& Reeder, D. M. (eds): Mammal species of the world: a taxonomic and geographic reference. 3rd ed. Johns Hopkins University Press, Baltimore, 2142 pp.

Trizio, L., Crestannello, B., Galbusera, P., Wauters, A., Tosi, G., Matthysen, E. \& Hauffe, H. C. (2005) Geographical distance and physical barriers shape the genetic structure of Eurasian red squirrels (Sciurus vulgaris) in the Italian Alps. Molecular Ecology 14: 469-481. doi: 10.1111/j.1365-294X.2005.02428.x

Wauters, L. A. \& Martinoli, A. (2008) Sciurus vulgaris. Pp. 349-360. In: Amori, G., CoNTOLI, L. \& NAPPI, A. (eds): Mammalia II, Erinaceomorpha, Soricomorpha, Lagomorpha, Rodentia, Fauna d'Italia. Vol. 44. Edizioni Calderini de Il Sole 24 Ore, Milano.

Revised version received May 21, 2014, accepted August 27, 2015, published October 19, 2015 